\title{
La Retórica Jurídica y el Derecho Penal
}

Legal Rhetoric and Criminal Law

\author{
Dennis Valeria Encalada González \\ Investigadora Jurídica, Equimacon S.A.
}

Artículo Original (Revisión)

RFJ, No. 3, 2018, pp. 215-235, ISSN 2588-0837

El texto principal se encuentra en italiano

RIASSUNTO: la seguente investigazione ha la finalità di analizzare l'origine del rapporto tra la retorica e il Diritto Penale; di fatto, la retorica o anche chiamata "l'arte della persuasione" dove non è necessaria la scrittura, fu introdotta da Socrate, un importante filosofo e maestro greco vissuto in Atene negli anni 300 a.C.. Si intrinseca con il Diritto Penale poiché il suo materiale normativo non è perfettamente codificato; infatti, la retorica costituisce una sorta di strumento mediante il quale un cittadino può sostenere la sua propria opinione sia nel ambito politico che giudiziario dinanzi, in questo caso, a un processo penale presentando accertamenti del fatto e un'ottima narrazione con il fine di ottenere il consenso della giuria. È di grande interesse menzionare, anche, la importanza di identificare le differenze e uguaglianze tra la retorica e le considerazioni di ordine giuridico; individuare le motivazioni principali per cui il Diritto Penale necessita della retorica per poi confrontarlo con il Diritto Penale ecuadoregno; esaminare le strategie utilizzate "dall'arte della persuasione" per comprovare i benefici e gli svantaggi che apporta nel Diritto Penale; infine, esporre alcune osservazioni per diminuire questa problematica trovando un equilibrio nella pratica della retorica.

PAROLE CHIAVI: retorica, logica, diritto, processo penale, razionalità.

ABSTRACT: the following investigation aims to analyze the origin of the relationship between rhetoric and Criminal Law; in fact, the rhetoric or also called "the art of persuasion" where writing is not necessary, was introduced by Socrates, an important Greek philosopher and teacher who lived in Athens in the 300 BC. It is intrinsic to Criminal 
Law since its normative material is not perfectly codified; in fact, rhetoric constitutes a sort of instrument by which a citizen can support his own opinion both in the political and judicial sphere before, in this case, a criminal trial presenting findings of the fact and an excellent narration in order to obtain the jury's consent. It is also of great interest to mention the importance of identifying the differences and equations between rhetoric and legal considerations; identify the main reasons why the Criminal Law requires rhetoric and then compare it with the Ecuadorian Criminal Law; examine the strategies used by "the art of persuasion" to prove the benefits and disadvantages it brings in Criminal Law; finally, to present some observations to reduce this problem finding a balance in the practice of rhetoric.

KEY WORDS: rhetoric, logic, right, criminal process, rationality.

RESUMEN: la siguiente investigación tiene la finalidad de analizar el origen de la relación entre la Retórica y el Derecho Penal; de hecho, la retórica o, también, nombrada "el arte de la persuasión" donde no es necesario la escritura, fue introducida por Sócrates, un importante filósofo y maestro griego subsistido en Atenas en los años 300 A.C. Se relaciona con el Derecho Penal, ya que, su material normativo non está perfectamente codificado; por consiguiente, la retórica constituye un instrumento, mediante el cual un ciudadano puede sustentar su propia opinión sea en el ámbito político que en el judicial frente, en este caso, a un proceso penal presentando acertamientos del hecho y una óptima narración con el fin de obtener el consentimiento del jurado. Es de grande interés mencionar, además, la importancia de identificar las diferencias y semejanzas entre la retórica y las consideraciones de orden jurídico; individuar la motivaciones principales por los cuales el Derecho Penal necesita de la retórica y luego confrontarlo con el Derecho Penal ecuatoriano; examinar las estrategias utilizadas por "el arte de la persuasión" para comprobar los beneficios y las desventajas que alteran el Derecho Penal; en fin, proponer algunas observaciones para disminuir este problema con la finalidad de encontrar un equilibrio en la práctica de la retórica.

PALABRAS CLAVES: retórica, lógica, Derecho, proceso penal, racionalidad. 


\section{INTRODUZIONE}

${ }^{159} \mathrm{Nel}$ Diritto Penale questa forma di oratoria giudiziale è essenziale quando, durante il processo penale, l'accusato cerca di convincere de la sua innocenza dinanzi al giudice e/o la giuria. Codesta relazione ha origini molto antiche, di cui si riconoscono due tappe: Atene Classica ( V - VI secolo a.C.) e Antica Roma ( II secolo a.C.). Si faranno maggiori accertamenti nelle seguenti pagine grazie ai lavori realizzati in passato da studiosi del Diritto, dei quali, la maggior parte sono italiani giacché la loro giurisdizione deriva direttamente dal Diritto Romano, anche se presenta alcuni cambiamenti; poiché, la retorica ha avuto un ruolo importante nel sistema processuale romano e di conseguenza ha determinato l'ordinamento giuridico di questo paese. Si menzioneranno: Alessandro Traversi, Maurizio Mazin, Carofiglio e Artosi; come si può intuire, sono numerosi gli autori specializzati in questo tema pero, a causa, del poco tempo e poco spazio a disposizione si prenderanno in considerazione solo alcuni di essi, in più, la maggior parte sono docenti universitari che insegnano la materia logica $e$ argomentazione giuridica, di fatto, nella facoltà di giurisprudenza delle università presenti in tutta Italia si insegna questa disciplina.

Nella presente investigazione si pretende conseguire una diagnosi delle ipotetiche soluzioni dei conflitti causati dal non limitare la retorica all'interno di un processo penale, attraverso l'uso di una metodologia attiva, esplorativa e apprensiva. Nei seguenti capitoli si parlerà della classificazione della retorica secondo la materia trattata, le tecniche utilizzate e si tenterà di dare una definizione che racchiuda tutte le informazioni ottenute, per rispondere alla seguente domanda: Cosa si intende per retorica?; successivamente, si argomenterà sul concetto di

159 Prima di iniziare lo svolgimento dell'articolo "La retorica e il Diritto Penale" è fondamentale ringraziare il Dottor Rubén Mendez, professore della Pontifica Università Cattolica dell' Ecuador, per le sue lezioni approfondite e meticolose sui vari argomenti adiacenti alla materia di logica giuridica; di fatto, hanno scaturito l'interesse di approfondire i due temi trattati in questo articolo, poiché, dato il poco tempo a disposizione delle ore di lezione del docente Mendez non è possibile analizzare dettagliatamente tutti i temi presenti nel programma universitario, ciò provoca da parte degli studenti una partecipazione "singola", cioè, lo studio individuale. Per questa ragione, si ritiene che il risultato finale di questa investigazione avrà un ruolo decisivo nella materia di logica giuridica, dal momento che, si tenterà di individuare i principali problemi tra la retorica e il Diritto Penale e, nello stesso tempo, le risposte più concrete per la sua soluzione. 
Diritto Penale, processo penale e le motivazioni per cui ha un legame molto stretto con l'oratoria giudiziale, con il fine di rispondere alla domanda: Quali sono le ragioni che provocano una interdipendenza tra il Diritto Penale e la retorica giuridica?. Quando si parla di Diritto Penale si fa riferimento, soprattutto, al processo penale poiché è lì che si introduce il tema "dell'arte della persuasione".

Nel corso dello svolgimento dell'articolo si ragionerà dei principali problemi, giacché, l'aspirazione finale è conseguire resultati utili che ci permetta l'accesso a maggiore informazione sulla relazione dei due temi trattati e in un domani poter prendere decisioni con il fine di migliorarla, in fine, capire la relazione tra retorica e la materia di logica giuridica.

Da non sottovalutare l'importanza di attribuire la definizione adeguata ai concetti giuridici verosimili come argomentazione giuridica e persuasione che hanno una propria descrizione, affinché, si possa avere una idea chiara dei temi trattati ma, particolarmente, poter raggiungere gli obiettivi stabiliti anteriormente.

\section{LA RETORICA}

Innanzitutto, il concetto di retorica è molto ampio e complicato, di conseguenza è necessario fare una piccola premessa che ci permetta sottolineare gli elementi più essenziali. Per Retorica si intende la capacità di persuadere qualcuno di qualcosa in specifico mediante l'analisi degli argomenti del tema in questione per poter individuare e sfruttare i punti di forza, può riguardare varie materie come: la medicina, la politica, la scienza e specialmente nell'ambito giudiziario, deliberativo ed epidittico ${ }^{160}$. Dunque, la definizione più prossima è la seguente: la retorica è la facoltà di scoprire in ogni argomento ciò che è in grado di persuadere, di cui si identificano le tecniche ed extra tecniche, quest'ultime possono essere testimonianze, documenti scritti o confessioni obbligate cioè l'insieme di elementi preesistenti che sono necessarie per conseguire le prime, in cambio, le tecniche

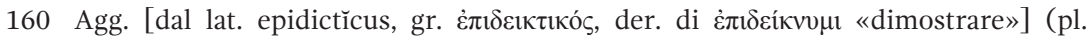
m. -ci). - Dimostrativo, espositivo; nella retorica antica, eloquenza e., il genere di eloquenza (per i Latini genus demonstrativum) usato dagli oratori greci nelle feste, nelle pubbliche cerimonie, nelle commemorazioni dei morti per la patria. Vocabolario Treccani. 
sono quelle che si ottengono attraverso la opinione, conoscimento e deduzione di un soggetto.

L'uditorio è il destinatario del discorso, ovvero, a chi si vuole persuadere, ciascuno dei quali possiede delle competenze, credenze ed emozioni; su queste basi, il soggetto che crea il discorso deve adattarlo secondo il tipo di auditorio.

È di utile importanza trascrivere direttamente l'intera definizione di Retorica secondo il vocabolario Treccani (un dizionario utilizzato da professionali nello studio di alcuni termini) dato le numerose difficoltà di capire i diversi concetti con elementi quasi similari, perciò, nel linguaggio professionale si intende: "L'arte del parlare e dello scrivere in modo ornato ed efficace; sorta nella Grecia antica con i sofisti, con finalità prevalentemente pragmatiche, come tecnica del discorso teso a persuadere ( $\mathrm{fu}$ quindi applicata all'oratoria giudiziaria), si viene successivamente ampliando nell'età classica e poi medievale e rinascimentale a tecnica del discorso sia orale sia scritto, con finalità anche estetiche, secondo un sistema di regole in cui fu organizzata dapprima da Aristotele, poi dalla trattatistica latina d'età classica (Cicerone, Quintiliano) e tarda (Marziano Capella, Boezio). Nel medioevo la retorica, la grammatica e la dialettica (la quale ultima ha per scopo la dimostrazione non la persuasione) costituivano le tre arti liberali (arti del trivio, in lat. artes sermocinales), distinte dalle quattro arti reali (arti del quadrivio, in lat. artes reales), l'aritmetica, la geometria, la musica e l'astronomia. Dopo una progressiva decadenza nell'età moderna, dovuta alla rivalutazione dei contenuti espressivi, la retorica ha riacquistato uno spazio rilevante nella linguistica e nella critica letteraria contemporanea, come teoria dell'argomentazione e come analisi delle realizzazioni lessicali, grammaticali e stilistiche, di testi scritti o anche orali, e delle loro funzioni espressive" ${ }^{161}$. Dall'altra parte, secondo il filosofo greco, Aristotele, la retorica si basa nell'osservazione empirica di cui alcuni uomini senza volere o non possono rispondere alla logica o alla dialettica; come la dialettica è, assai, lontana dalla logica formale causato dal fatto che si cerca incessantemente la opinione per iniziare una discussione, mentre, la retorica è lontana dalla dialettica poiché si cerca discutere dinanzi a un soggetto di poca valore che prende in considerazione temi non troppo aderenti all'argomento centrale.

161 Vocabolario Treccani. 


\section{Bisogna fare un chiarimento sul termine dialettico ${ }^{162}$ che si definisce}

162 "1. Arte del dialogare, del discutere, come tecnica e abilità di presentare gli argomenti adatti a dimostrare un assunto, a persuadere un interlocutore, a far trionfare il proprio punto di vista su quello dell'antagonista: un oratore dalla d. stringente; ridurre al silenzio l'avversario con la propria d.; con senso peggiorativo, modo sottile e capzioso di argomentare: con la sua d. ci metterebbe tutti nel sacco. 2. Nel linguaggio filosofico, il termine ha avuto accezioni diverse, secondo le epoche e le scuole. a. Nel pensiero antico, è in genere l'arte dialogica, come metodo di dimostrazione mediante brevi domande e risposte (adoperato da Socrate in contrapp. ai lunghi discorsi dei sofisti). In Platone, è sia il processo interiore che conduce ai concetti più generali e ai principî primi della realtà intelligibile, sia l'arte di dividere le cose in generi e specie, di classificare in concetti per meglio progredire nell'analisi. In Aristotele, parte della logica, intermedia tra l'analitica e la retorica, che studia le forme argomentative imperfette, da cui si traggono conclusioni soltanto probabili e non rigorosamente necessarie. b. Nel pensiero medievale, la dialettica s'identifica con la logica e diviene una delle arti del trivio, insieme con la grammatica e la retorica. c. In Kant, d. trascendentale o «logica dell'apparenza» è la teoria degli errori naturali dello spirito umano, che si illude di poter determinare, sul solo fondamento di ragionamenti teorici, la natura dell'anima, del mondo e di Dio, e cade invece in inevitabili, inestricabili contraddizioni. Nell'idealismo post-kantiano e per Hegel in partic., la dialettica è la natura stessa del pensiero che si sviluppa secondo proprie leggi ma in modo conforme allo sviluppo della realtà anzi rappresentandone la struttura stessa; è quindi movimento e sviluppo che da un concetto astratto e limitato (affermazione o tesi) passa al suo opposto (negazione o antitesi) per giungere a una sintesi (negazione della negazione) che conserva elementi fondamentali dei precedenti e opposti momenti, i quali peraltro non sarebbero mere astrazioni concettuali bensì pensieri concreti, determinazioni storiche, effettive formazioni culturali, sociali, ecc. Nel pensiero di Marx tale movimento si specifica e si concretizza come sviluppo dell'antagonismo tra classi sociali contrapposte e come relazione di opposizione tra forze produttive e rapporti di produzione. In Croce, infine, si distingue una d. dei distinti, per cui lo spirito, secondo un processo circolare, passa da un grado all'altro senza annullare i precedenti, e una d. degli opposti che, nella sfera delle cose concrete, opera la sintesi della tesi e dell'antitesi. 3. Nel pensiero moderno, e anche nel linguaggio com. (in diretta connessione con le accezioni che il termine ha avuto nel pensiero filosofico), il processo risultante dalla lotta o dal contrasto di due forze: uno storico cui non sfugge la complessa d. tra cultura e politica; o, più spesso, gioco di forze contrastanti che collidono e si ricompongono incessantemente: la d. delle forze economiche; la d. dei partiti. Il termine è inoltre usato (per es. nel linguaggio della critica) per indicare quell'argomentazione che giustappone idee opposte o contraddittorie e generalmente tende a far giungere tale conflitto a un qualche esito, che si presume necessario e inevitabile". Vocabolario Treccani. 
della seguente forma: chiamato, anche, l'arte del dialogare di cui il suo punto di forza è l'abilità di porre gli argomenti più opportuni con il fine di provare un assunto. Per quanto riguarda la logica, essa è una disciplina che ha l'obiettivo di studiare le condizioni di validità delle argomentazioni deduttive, le sue origini risalgono con l'elaborazione della dottrina delle forme nelle quali si muove, ragionando, il pensiero; codesta fu scritta da Aristotele, il quale in un principio si riferisce ad essa con il termino "analitico" e, con il passare del tempo, con l'aiuto dei pensieri e conoscimenti di personaggi illustri questo termino ha subito cambiamenti fino ad essere ciò che oggi.

Ritornando, alla retorica è fondamentale focalizzarsi nella sua origine e mutazione nel corso del tempo, tra cui, si sottolineerà l'importanza di alcune tappe storiche, affinché, si possa comprendere il nesso con il diritto.

\subsection{Origine e tappe storiche della Retorica.}

"Nel corso di nove secoli, la retorica ha plasmato, in diversi modi, la vita spirituale di greci e romani" ${ }^{163}$. La Retorica ha origini molte antiche, di fatto, viene introdotto per la prima volta da Aristotele, un importantissimo filosofo greco vissuto in Atene tra la fine degli anni 300 a.C. e l'inizio degli anni 400 a.C.. Per lui la retorica riguarda, più che altro, la forma speciale di pensare che è limitato nel momento in cui si applica. Questo filosofo fu alunno dell'Accademia di Platone che a sua volta è stato alunno di Socrate; attualmente, molti lo considerano il primo investigatore scientifico, poiché, le sue opere filosofiche e teologiche hanno avuto un ruolo predominante nei conoscimenti odierni. Come tutti sappiamo, la civiltà greca è una delle più importanti sia nell'ambito filosofico, teologico che politico; infatti, la loro organizzazione sotto questo punto di vista era ottimo, dal momento che le città greche erano indipendenti le quali prendevano il nome di polis ${ }^{164}$ formato da un gruppo di persone che avevano la funzione di prendere decisioni autonomamente.

Le più importanti polis erano quelle di Atene e Sparta, in un primo momento erano governate da un re poi dall'aristocrazia, a partire del 508 a.C. in Atene tutti i cittadini partecipavano alla vita politica e per

163 Ernzt Robert Curtius. 1955.

164 Città-stato. Vocabulario Treccani. 
questa ragione fu istituita l'assemblea del popolo chiamata Ecclesia; fu così che apparve la prima forma di governo, ovvero, la Democrazia ${ }^{165}$. Sia gli organi legislativi che gli organi giudiziari sono totalmente nelle mani dei cittadini ${ }^{166}$, da non confondere con popolazione ${ }^{167}$ poiché essa era costituita da greci e schiavi (prigionieri di guerra, i quali non possedevano nessun tipo di diritto), con lo scopo di consolidare il potere del popolo. Un importate fattore che caratterizza questa prima forma di giurisdizione è l'assenza della figura del giurista (avvocato), non c'era nessuno che potesse studiare e approfondire la disciplina del Diritto e, soprattutto, nelle situazioni di conflitti di materia civile o penale i soggetti immischiati dovevano difendersi da soli utilizzando la parola per convincere la giuria composta a sua volta da altri cittadini, è qui che Arsitole elabora il concetto di Retorica e della sua importanza nei Tribunali.

Con il passare del tempo, un'altra civiltà è riuscita ad emergere fino ad arrivare ad essere una dei più grandi Imperi dell'Occidente: Impero Romano (27 a.C. -476 d.C.). In origine, questa civiltà era composta da contadini e agricoltori. Si distinguono tre divisioni dell'epoca romana: Monarchia, Repubblica e Impero. Durante le due ultime tappe, i romani entrarono in contatto con i greci, è lì dove, si inspirano per creare la propria cultura e organizzare il sistema politico. Tuttavia, si riconosce lo spicco della Retorica nel sistema processale ${ }^{168}$ durante la epoca

165 Forma di governo che si basa sulla sovranità popolare e garantisce a ogni cittadino la partecipazione in piena uguaglianza all'esercizio del potere pubblico. Vocabolario Treccani.

166 È colui che possiede la cittadinaza: Condizione di appartenenza di un individuo a uno Stato, con i diritti e i doveri che tale relazione comporta; tra i primi, vanno annoverati in particolare i diritti politici, ovvero il diritto di voto e la possibilità di ricoprire pubblici uffici; tra i secondi, il dovere di fedeltà e l'obbligo di difendere lo Stato, prestando il servizio militare, nei limiti e modi stabiliti dalla legge. Vocabolario Treccani.

167 L'insieme di individui o oggetti in un determinato ambito, considerati nel loro complesso e nell'estensione numerica. Enciclopedia Treccani.

168 4. a. Nel linguaggio giur., complesso delle attività e delle forme mediante le quali vengono risolte le controversie tra due soggetti da un terzo imparziale e disinteressato (il giudice), oltre che dotato del potere effettivo di imporre con autorità alle parti l'accettazione della sua sentenza: la storia del diritto ha progressivamente formalizzato e legalizzato la dinamica del processo fino a farne il momento emblematico e riassuntivo dell'intera esperienza giuridica: p. civile, penale, amministrativo, secondo la natura della controversia; p. di cognizione, di esecuzione; fare, celebrare, istruire, aprire, chiudere un p.; essere, mettere, 
Repubblicana, infatti, l'accusato mediante le tecniche di retorica mira a smuovere le emozioni e screditare la parte contraria. Un personaggio si distingue rispetto ad altri, Marco Fabio Quintiliano, grazie a uno studio approfondito di 20 anni sulla retorica; molti sono stati i suoi alunni, tra cui, è necessario ricordare i nipoti dell'Imperatore Domiziano ed altri filosofi. La sua fama si rimembra tutt'oggi per merito di un manoscritto nominato "Istituto Oratoria", dove si stabilisce la miglior forma d'istruzione per i giovani; un aspetto fondamentale di Quintiliano è la sua professione come avvocato, infatti, si può affermare che per la prima volta viene introdotto lo studio della retorica nel diritto , giacché, ci sono due punti di vista, cioè, la parte filosofica della struttura dell'arte della persuasione e la sua funzione nel sistema processuale.

Qualche secolo dopo, nel Medioevo, il concetto di Retorica viene ripreso nuovamente in Francia, a quell'epoca chiamata Gallia, in cui questa forma di oratoria ha un ruolo importante rispetto agli altri stili. In Europa, la situazione economica permise l'origine di un nuovo ceto sociale, la borghesia, composto per lo più da commercianti che anno dopo anno prese sempre più potere all'interno della società che nell'ambito politico. Gli intellettuali, con maggior fama, che si dedicarono all'osservazione della lingua e le sue varie sfaccettature furono: Bernardo di Chiaravalle, Venanzio Fortunato, Sidonio Apollinare e Marbodo di Rennes.

Finalmente, nel '600 e '700, la contribuzione di molti acculturati ha permesso giungere a una più complessa definizione della struttura del

mandare sotto p., essere sottoposto, o sottoporre qualcuno, a un procedimento penale. In partic., p. accusatorio, forma di processo penale marcatamente garantista, tipico degli ordinamenti anglosassoni e introdotto, anche se non integralmente, nell'ordinamento italiano dal nuovo cod. proc. pen. del 1988, caratterizzato dall'assoluta parità di poteri processuali riconosciuti all'accusa e alla difesa; p. inquisitorio, forma di processo penale, tipica degli ordinamenti giuridici dell'Europa continentale, caratterizzata da una spiccata asimmetria tra i poteri processuali della difesa e quelli dell'accusa a vantaggio di quest'ultima, specie nella fase di ricerca delle prove da portare in giudizio. In usi estens. e fig.: non vorrai farmi un p. se ho tardato un po'!; non andrò sotto p. per aver perso il tuo libro, e sim., con riferimento a comportamenti eccessivamente severi e inquisitorî; fare il p. alle intenzioni, voler giudicare una persona non dai fatti obiettivi ma dalle intenzioni che, a ragione o a torto, le si attribuiscono. b. estens. Incartamento processuale: esaminare, studiarsi il processo. c. Nell'uso com., dibattimento pubblico, udienza: assistere a un p.; il p. è rinviato. Enciclopedia Treccani. 
tema in questione ma, soprattutto, con la nascita di nuove discipline e di nuove professioni come nell'ambito: giuridico, amministrativo e didattico in relazione all'educazione; si osserva e si definisce il legame tra l'arte della persuasione e il diritto penale.

Un nuovo concetto che emerge è il nesso tra logica e retorica che si analizzerà nel seguente capitolo come una delle tante tecniche che permettono una corretta formulazione della Retorica, in più, gli elementi antecedenti e finali necessari per facilitare il processo.

\subsection{Tecniche utilizzate per perfezionare l'arte della persuasione ed altre caratteristiche.}

Per formulare una buona retorica, è essenziale regolare la comunicazione mediante i principi stabiliti da Beaugrande y Dressler: la efficienza del testo che abbia per lo meno un minimo di sforzo da parte dei soggetti interessati e che possa scaturire un'impressione appropriata in modo tale da produrre condizioni favorevoli per ottenere gli obiettivi comunicativi, in più, ci dev'essere coerenza tra il testo e l'ambiente circostante di questi soggetti. Inoltre, la comunicazione deve rispettare 7 criteri: la coesione, la coerenza, l'intenzionalità, l'accettabilità, la situazionali, l'informativa e l'intertestualità. Il primo criterio organizza gli elementi grammaticali; il secondo fornisce un significato semantico ovvero il significato del discorso; il terzo è determinato dall'obiettivo prefissato dal soggetto che compone un testo, ciò, che vuole trasmettere; il quarto è la risposta del ricevente; il quinto, ai cambiamenti relazionati al contesto esteriore; il sesto, in cambio, è il grado di interesse che possiede l'informazione e, infine, il settimo che ha come fine collegare il tipo di testo ad un altro.Il discorso ${ }^{169}$ è la rappresentazione verbale che può essere orale o scritta

169 1. a. L'atto del discorrere, dell'esprimere il pensiero per mezzo della parola: lasciare in tronco un d.; perdere il filo del d.; discorsi senza importanza; d. ambiguo, sibillino, sciocco, scipito; fare discorsi a vanvera, senza capo né coda. b. L'argomento su cui si discorre: cambiare, mutare d.; tocca sentire certi d.!; questo è un altro d., anche con sign. estens., è un'altra cosa. c. Colloquio, conversazione: attaccare d.; venire, entrare in d.; è meglio troncare questi d.; il d. cadde sul teatro; d. amichevole, alla buona. d. Al plur., e in tono più o meno spreg., parole, chiacchiere: pochi d.!; non facciamo troppi d.!; senza tanti d., senza andare per le lunghe, con franchezza; e contrapposto ai fatti: questi sono d.!; tutti d.!; tutti bei discorsi! 2. Trattazione ordinata e diffusa intorno a qualche preciso argomento (di solito pronunciata in pubblico, ma che può anche 
ed è formata da una o più frasi coordinate tra di loro, in modo tale, da ottener un senso logico; esso, ha distinte fasi che corrispondono alla creazione di una idea, poi, la progettazione di uno schema, la redazione utilizzando un linguaggio chiaro e vivace, per finire, l'espressione che giova di un ruolo predominante.

Anticamente, si avvaleva spesso dell'auxeis e tutt'ora si utilizza, però possiede un nome completamente distinto, si relaziona alla parola crescere o aumentare, giacché, ha il fine di storcere la realtà peggiorando la situazione.

La logica ${ }^{170}$ è un alto elemento essenziale per iniziare tutto questo processo, essa è la disciplina che studia le condizioni di validità delle

essere soltanto scritta): preparare, scrivere, comporre un d.; fare, tenere, pronunciare un d.; improvvisare un d.; sentire, ascoltare un d.; d. politico; d. accademico; d. d'inaugurazione, di chiusura, ecc.; d. lungo, noioso, prolisso, monotono, ampolloso, risoluto; la pubblicazione dei d. di Cavour. D. della corona, quello del sovrano dinanzi al parlamento all'inizio di ogni legislatura, nel quale si traccia il programma politico e amministrativo del governo. 3. a. In grammatica, parti del d., le varie categorie nelle quali la grammatica tradizionale suole dividere il corpo lessicale di una lingua, in base alla funzione che le singole parole adempiono nella frase. In italiano si distinguono in genere nove parti del discorso, delle quali cinque sono dette variabili, in quanto hanno una flessione (sostantivo, aggettivo, articolo, pronome, verbo), e quattro invariabili (avverbio, preposizione, congiunzione, interiezione). b. In sintassi, con riferimento al modo con cui, narrando o scrivendo, si riproducono le parole dette da altri, si distingue un d. diretto, in cui le parole sono riprodotte nella forma originale (per es.: «Mi disse: - Tornerò domattina»), e un d. indiretto, quando esse sono riferite nella forma voluta dalle leggi della dipendenza sintattica (per es.: «Mi disse che sarebbe tornato l'indomani mattina»). Si distingue infine un tipo intermedio, detto d. indiretto libero, in cui le parole o il pensiero di una persona vengono riferiti dall'autore in forma indiretta, ma tacendo il verbo dichiarativo reggente o ponendolo in inciso, come se si avesse la citazione diretta; per es.: «Di tutte queste riflessioni nulla trasparì sul viso di Tereso. Anche lui, egli disse ad un tratto come ricordandosi, aveva da sbrigare insieme con il segretario certe faccende che non tolleravano di essere rimandate. Si sarebbero dunque veduti la sera» (Moravia, La mascherata. 201).

170 3. a. Nell'uso com ., l'arte di condurre il ragionamento, l'argomentazione, il discorso in modo che le idee siano tra loro connesse e si sviluppino con razionale procedimento l'una dall'altra; quindi, giustezza di ragionamento: condurre una dimostrazione con logica, con 1. perfetta, serrata, sottile, severa, inesorabile; discorso pieno di l., o privo di l., senza l.; procedimento a filo di logica; e in quanto il ragionamento si estrinseca nelle azioni: condotta, comportamento, risoluzione 
argomentazioni deduttive (dal macro al micro), dunque, l'essere umano ragiona prima di parlare o scrivere un testo; pensare permette individuare e organizzare le idee in modo tale che una volta espresse, abbiano un significato con un alto grado di eloquenza; inoltre, la capacità di ragionare può essere di grande aiuto.

Due fattori che hanno la parola decisiva sull'efficienza della retorica formulata, sono le emozioni e l'uso di tecniche della disciplina della psicologia; infatti, per persuadere i destinatari è necessario catturare la loro attenzione e saperli "manipolare" con il fine di raggiungere la loro parte interiore e smuoverli; codesto si fa, anche, in ambiti molto seri come nel Diritto con buoni risultati, tuttavia, non si dovrebbero verificare questi tipi di situazione poiché si dovrebbe mantenere sempre e comunque l'obiettività, ed è qui che sorge il problema del tema preso in considerazione.

Questi insieme di concetti, "formano" la Retorica, vale a dire che seguendo passo per passo i criteri menzionati prima e avendo le idee chiare sui diversi significati di alcuni elementi presi in considerazione si può conseguire un ottimo risultato. Altri aspetti fondamentali sono le figure, vale a dire, i tipi di espressioni non comuni al linguaggio parlato che hanno lo scopo di attirare l'attenzione del destinatario o destinatari.

\section{ORDINAMENTO GIURIDICO}

L'ONU (Organizzazione delle Nazioni Unite) riconosce la presenza di 206 paesi nel pianeta Terra, ciascun dei quali possiede un proprio Ordinamento Giuridico; esso è differente tra stato in stato dovuto agli antecedenti storici, culturali e religiosi. L'Ordinamento Giuridico $\mathrm{o}$, anche, riconosciuto come Diritto è l'insieme di norme che hanno l'obiettivo di regolare le relazioni tra gli individui di una Società all'insegna della pace, dell'armonia e della tranquillità. Uno dei fattori

privi di logica. Per la l. deontica, v. deontico. b. Più genericam., modo di ragionare: bella l. la tua!; ha una 1. tutta sua particolare. c. fig. La ragione che è alla base di un determinato comportamento e che lo giustifica (anche con riferimento ad atti pubblici e a decisioni o scelte politiche). Con altro uso fig., la 1. dei fatti, degli avvenimenti, il modo con cui essi sono concatenati, procedendo di necessità l'uno dall'altro; analogam., la l. della storia, la l. interna d'un sistema linguistico, e simili. d. In informatica, l'insieme dei principî scelti per la progettazione di un sistema di elaborazione automatica di dati. Vocabolario Treccani. 
principali del Diritto è la giustizia, di fatto, queste norme che possono essere permissive, proibitive e precettive sono emanate per risolvere ipotetici conflitti garantendo giustizia ai soggetti implicati.

Da tutto ciò, si può dedurre che la disciplina del Diritto è molto ampio, giacché, sono molteplici gli ambiti a cui è indirizzato; infatti, questa materia e la realtà sociale sono strettamente connesse e si possono distinguere diverse ramificazioni.

Ci sono tre concezioni sull'O.g., la teroria normativa (H. Kelsen), la teoria istituzionale (S. Romano) e la teoria del rapporto (A. Levi), la particolarità di tutte e tre è relativo al fatto che si uniscono. Bisogna ricordare che il Diritto è oggettivo, vale a dire che si considera solo la parte obiettiva di qualsiasi situazione con il fine di conseguire una giustizia equa.

Principalmente, ci sono due grandi suddivisioni del Diritto ${ }^{171}$ dal punto di vista dell'intervenzione dello Sato: il Diritto Pubblico e il Diritto Privato. Il D.Pu. regola tutte le relazioni tra i particolari e lo Stato, mentre, il D.Pr. regola i rapporti tra privati; a sua volta, ciascuno dei due diritti possiede altre classificazioni. Nel D.Pr. si distinguono secondo le materie trattate come: il D. Costituzionale, il

171 2. a. Complesso ordinato di norme (specificamente, nel linguaggio giur., d. oggettivo), variabili da tempo a tempo e da popolo a popolo, che prescrivono o vietano determinati atti e comportamenti, con lo scopo di regolare i fondamentali rapporti (familiari, economici, politici) su cui si regge l'organizzazione, la convivenza e la sopravvivenza della società, e disciplinare le relazioni intersoggettive, stabilendo insieme le sanzioni e gli altri mezzi adatti a impedire le azioni più dannose per il gruppo sociale e a prevenire o risolvere i conflitti e le tensioni che possono minacciare l'ordine, la pace e la sussistenza stessa del gruppo: i fondamenti, i principî, il concetto del d.; la codificazione del d.; il d. romano, il d. germanico, il d. intermedio; il d. vigente in Italia; d. positivo, l'insieme delle norme che costituiscono l'ordinamento giuridico di un paese (contrapp. in genere al $\mathrm{d}$. naturale: v. naturale, n. 2 a e giusnaturalismo). Per i varî rami del diritto oggettivo, e cioè d. pubblico e privato, d. interno e internazionale, d. civile e penale, e inoltre d. agrario, amministrativo, canonico, commerciale, costituzionale, ecclesiastico, fallimentare, industriale, d. del lavoro, d. della navigazione, e d. processuale, si veda ai singoli aggettivi o sostantivi determinanti. b. Scienza che ha per oggetto lo studio di tali norme nel loro insieme o nei loro particolari raggruppamenti: scuole, cultori di d.; professore di d. (e, anche in questo sign., d. civile, d. penale, ecc.); d. comparato (v. comparato). Vocabolario Treccani. 
D. Penale, il D. Processuale, il D. Amministrativo e il D. Tributario. Nel D.Pu. è composto dal D. Civile e il D. Commerciale, nonostante la classificazione, esistono alcune che appartengono tanto al Diritto Pubblico che al Diritto Privato.

Il Diritto Penale disciplina i fatti costituenti reato, le norme che compongono i Codici Penali individuano gli ipotetici casi di reato e stabiliscono, anche, le sanzioni che sono di varia natura secondo il crimine commesso. Per reato si intende l'azione che va contro una legge e che può recare danni ad un altro soggetto; per prevenire questi reati, il Diritto Penale si avvale di un strumento, ovvero, le sanzioni penali; inoltre, questi tipi di crimini si basa su tre principicardine: il principio di materialità, il principio di necessaria lesività o offensivista e il principio di colpevolezza. Il processo penale avviene dopo la "notizia di reato" che si ottiene mediante la denuncia ${ }^{172}$, il referto (medico) e la querela ${ }^{173}$, successivamente, si esercita l'azione

172 1. In diritto penale, atto formale informativo, facoltativo o obbligatorio, con il quale si dà notizia alla competente autorità di un reato perseguibile d'ufficio: sporgere, presentare, inoltrare una denuncia. 2. a. Dichiarazione, notificazione richiesta da una norma di legge per determinati scopi (di stato civile, igienici, fiscali, ecc.): d. di una nascita, di un decesso; d. sanitaria, atto, facoltativo o obbligatorio a seconda dei casi, con cui un sanitario informa l'autorità competente (medico provinciale, ufficiale di stato civile, di pubblica sicurezza, ecc.) di fatti che essa ha interesse a conoscere e che riguardano l'esercizio professionale; d. amministrativa, dichiarazione con la quale il privato porta a conoscenza della pubblica amministrazione la illegittimità o inopportunità di un atto amministrativo al fine di sollecitare l'esercizio dei poteri di annullamento e di revoca di ufficio; d. dei redditi, lo stesso che dichiarazione dei redditi. b. Denominazione tradizionale (come traduz. del termine lat. nunciatio) di alcune azioni giudiziarie a tutela della proprietà o del possesso: d. di danno temuto, d. di nuove opere, quelle mediante le quali l'interessato chiede all'autorità giudiziaria che vengano disposti gli opportuni provvedimenti per evitare i danni che egli teme possano derivare a un suo bene, rispettivamente, dalle particolari condizioni in cui si trova una qualsiasi cosa altrui (edificio, albero, ecc.) o da un'opera intrapresa da altri sul proprio o altrui fondo.

173 2. Nel diritto penale, atto con cui la persona offesa da un reato manifesta, nei casi espressamente previsti dalla legge, la volontà che il colpevole venga processato (costituisce una condizione di procedibilità, e pertanto, in sua assenza, l'azione penale contro il reo non può iniziare o continuare il suo corso): presentare, proporre, sporgere querela; dare querela a qualcuno; ritirare la q.; il reato è perseguibile a querela (o per q., meno bene su q.) di parte; reato estinto per remissione di querela; q. per ingiurie, per diffamazione, per violazione di domicilio, ecc. In partic., q. di 
penale una volta portati a capo le determinate investigazioni per sostenere l'accusa; durante, il processo il soggetto indagato ha l'occasione di difendersi individualmente e/o , attraverso un avvocato, nel primo caso la retorica utilizzata è di un livello mediocre mentre nel secondo caso la retorica è arricchita da termini giuridici fattibili, con l'intenzione di convincere la giuria e il giudice dell'innocenza dell'individuo implicato o a "ridurre" la sanzione. Capire ciascuna fase di un processo penale è difficile, giacché, è molto complesso; per questo motivo, si è cercato di fare un riassunto con le fasi principali senza i quali non può iniziare un processo penale.

Dopo tutto, come si è affermato anteriormente, il Diritto è diverso per ogni paese e, di conseguenza, anche le caratteristiche del processo penale e del Diritto Penale non sono omogenee, pertanto, analizzerò il sistema penale in Ecuador poiché comporta un fattore importante, del quale si parlerà nel seguente capitolo.

\subsection{Il Diritto Penale in Ecuador}

L'Ecuador, è un paese sudamericano appartenente al "Terzo Mondo" ${ }^{174} \mathrm{o}$, meglio dire, ai paesi in via di sviluppo; questa caratterista

falso, istanza processuale con cui l'interessato impugna, fornendo adeguate prove, un atto pubblico o una scrittura privata per ottenerne la dichiarazione giudiziale di falsità. Per q. di nullità, in diritto canonico, v. nullità. Pegg. querelàccia, querela che causa fastidî, o che può avere conseguenze penali di qualche gravità per la persona querelata. Vocabolario Treccani.

174 Denominazione entrata nel linguaggio delle relazioni internazionali alla vigilia della conferenza di Bandung del 1955, per indicare i paesi dell'Asia, Africa e America Latina, appena usciti dalla soggezione coloniale oppure in lotta per il conseguimento dell'indipendenza. Tale espressione è rimasta nell'uso per designare i paesi caratterizzati da un basso prodotto interno lordo pro capite, da una elevata crescita demografica e da una struttura produttiva fortemente dipendente dall'importazione di capitali e tecnologie dai paesi industrializzati; sono preferibilmente detti paesi in via di sviluppo. Per terzomondismo si intende l'atteggiamento favorevole ai paesi del T., che può manifestarsi sotto forma di solidarietà politica, di sostegno economico, di accentuato interesse culturale ecc. In particolare, con questo termine si è indicato negli anni 1960 e 1970 un orientamento politico, sviluppatosi soprattutto nell'ambito della nuova sinistra, di sostegno alle lotte di liberazione dal dominio coloniale o neocoloniale, ai movimenti rivoluzionari operanti nei paesi del T. e ad alcuni Stati come Cuba e la Cina. Enciclopedia Treccani. 
lo accomuna al resto degli Stati confinanti. La Giustizia equadoregna si caratterizza per l'introduzione di nuove idee che hanno scaturito l'interesse della maggior parte degli avvocati e futuri avvocati della nazione. La legislazione presente in questo paese ha origini molto antiche, infatti, proviene dal Diritto Romano, perciò si possono distinguere molte assomiglianze; il Codice Civile è l'esempio basico di questa tramandamento. In Ecuador è stato incorporato il Sistema di Giudizio Orale inspirato al ruolo della retorica nel sistema accusatorio orale molto efficace nell'Amministrazione Pubblica. Il Codice Organico Integrale Penale (COIP) afferma la presenza di un testimonio orale accompagnato da suoni fonici orecchiabili e chiari, un altro aspetto da prendere in considerazione è l'ambiente circostante, siccome influisce psicologicamente al testimonio giudiziale. Il Sistema Accusatorio Orale possiede i seguenti principi: oralità, contradizione, mediazione, concentrazione e pubblicità; una delle principali motivazioni per cui si applica questo tipo di Sistema è dovuto a ragioni scientifichetecniche, sociali, etiche e fisiche; tutto sommato, bisogna riconoscere anche l'epoca in cui ci troviamo, giacché, permette la piena efficienza del Sistema Accusatorio Orale.

Nel suo complesso, il sistema penale ecuadoregno non è dei migliori presenti rispetto a molti altri; basti pensare all'immagine che ha dal punto di vista internazionale, in più, la corruzione non giova a favore.

\section{CONCLUSIONI}

Prima di iniziare con l'ultimo capitolo, non per questo meno importante, dove si tratterà di risolvere e conseguire gli obiettivi generali e specifici stabiliti antecedentemente, è fondamentale sottolineare un aspetto inatteso nello svolgimento dell'articolo giuridico, ovvero, la marea di concetti e termini giuridici e non solo, trovati. Come si è visto nei primi capitoli che riguardano il concetto di Retorica è stato di vitale importanze avvalersi di un vocabolario professionale e academico, poiché, i termini relazioni a questo tema sono molteplici e abbastanza simili, come: retorica, dialettico, discorso, denuncia, querela e molti altri concetti. La Retorica è un tema molto amplio, racchiude in sé numerosi aspetti della disciplina linguistica come la grammatica, della razionalità ${ }^{175}$, le emozioni, la

175 Consiste nella riflessione attenta con il fine di conseguire benefici e non il contrario. In questo caso, l'uso della logica per persuadere un soggetto. 
psicologia e i gesti, infatti, quest'ultimo ha una funzione scenografica. Detto ciò, si procederà con lo studio del marco teorico.

Dalle informazioni raccolte si deduce che la Retorica Giuridica ha avuto un ruolo predominante nel corso della Storia in diversi ambiti; tuttavia, continua ad essere un elemento essenziale nel Diritto Penale, specialmente durante il processo. C'è stata una trasformazione radicale, ovvero, prima questo strumento di oratoria era elementare e non ben definito; con il susseguirsi degli anni, i fattori storici e culturali aiutarono a modellare il concetto di Retorica, la quale nacque solo per persuadere una giuria nell'Antica Grecia rimase intatte acquistando sempre piú potere. Una notevole distinzione tra quella "vecchia" e quella "moderna" riguarda la presenza di regole, di fatto, nel passato c'era solo bisogno di parlare ed il gioco era fatto mentre adesso è regolata, in modo tale, che continui ad avere la sua importanza pero subordinato ad altri temi.

Sebbene, conseguire l'obiettivo delle tecniche e fasi, seguite alla lettera, per la formulazione di una buona "persuasione" è facile, si riscontra un grande problema nell'ambito giuridico. Precedentemente, con l'analisi del concetto di Diritto, lo si è associato alla parola "obiettività"176, pertanto, le emozioni non dovrebbero essere prese in considerazione nel momento di giudicare o emettere una sentenzia perché in caso contrario si andrebbe contro la etica e non si farebbe giustizia. La soluzione migliore consiste nel individuare i possibili limiti che siano rispettati sia dai soggetti emittenti ${ }^{177}$

176 1. L'atteggiamento di chi vede e giudica persone, eventi, circostanze con realismo e imparzialità, ed è quindi esente da pregiudizî e da passioni personali: analizzare con o. una serie di fatti; giudicare con o. un'opera; la difficile o. dello storico; analogam., o. di un giudizio, di una critica; esporre l'accaduto con assoluta o., con scrupolosa obiettività. 2 . Il fatto di essere obiettivo, cioè vero o valido per se stesso, indipendentemente dal giudizio o dall'interpretazione che altri ne possa dare. Per es.: a. In fisica, principio (o assioma) di o., principio secondo il quale il comportamento di un sistema è indipendente dall'osservatore; (un'altra diffusa ed equivalente formulazione afferma che la descrizione fisica di un sistema non muta se si cambiano arbitrariamente il sistema di riferimento geometrico e l'origine dei tempi). b. Nel linguaggio medico, il complesso degli elementi clinicamente significativi che emerge dall'esame obiettivo del malato. Vocabolario Treccani.

177 2. s. m. e f. Nel linguaggio econ., colui che rilascia un pagherò cambiario e ne diviene così l'obbligato principale; in linguistica e in teoria della comunicazione, chi formula e invia un messaggio, la fonte di un'informazione. Vocabolario Treccani. 
che dai interlocutori, infatti, i secondi devono considerare solo le affermazioni accompagnate con prove e non farsi ingannare dall'uso eccessivo di parole eloquenti e la drammaticità dei gesti; quindi, i fattori di limitazione sono i seguenti: per ciascuna affermazione una prova, non utilizzare le emozioni come strumento di persuasione, non giocare con gli elementi psicologici e, per finire, usare la obiettività in qualsiasi dichiarazione.

Per concludere, è fondamentale porre la ragione prima dei sentimenti, soprattutto, quando riguardano tematiche giuridiche, poiché, è più che corretto avere e dare osservazioni eque ${ }^{178}$ ad entrambe le parti.

Questo articolo non solo ha permesso individuare il problema tra la Retorica Giuridica e il Diritto Penale, bensì, entrare a conoscenza del significato corretto di ciascuna parola; quindi, il lettore potrà interagire e arricchire il proprio vocabolario "mentale" con l'apprendimento di concetti verosimili a prima occhiata, però, con elementi completamente distinti gli uni dagli altri. L'importanza di sapere la differenza di codesti è sostanziale, soprattutto, per gli studenti della Facoltà di Giurisprudenza poiché in pochi anni saranno degli avvocati; attualmente, la domanda nel mondo del lavoro è abbondante, l'opposto dell'offerta, quindi lo studente deve eccellere rispetto ad altri ampliando i propri conoscimenti.

\section{REFERENZE}

A., A. (2016). Ragionamento giuridico. Torino: Giappichelli.

Banfi, A. (s.d.). La Civiltà Greca - Storia. Tratto da Oilproject: http:// www.oilproject.org/lezione/diritto-e-retorica-20134.html

178 Che è conforme a equità, cioè alla giustizia nel suo ampio significato morale: pretendere un'e. distribuzione delle ricchezze; non sarebbe e. dar tutto a lui; di persona, che ha equità: un giudice equo. In senso più materiale, giusto, umano, debitamente proporzionato: condizioni e., un e. compenso; per e. affitto, e. canone, v. canone, n. 4. Avv. equaménte, con equità, con giustizia, con giusta proporzione: giudicare equamente; ripartire equamente gli utili o gli oneri. 
Bearzot, C. (2007). Diritto e retorica nella democrazia. Etica \& Politica, 22.

Beaugrande, R. y Dressler, W. (1981). Introduction to Text Linguistics. London, NewYork: Longman

Bobgo. (2012). Filosofia - Forum filosofico sulla ricerca del senso dell'essere. Tratto da IL forum di iflesioni: https://www.riflessioni.it/ forum/filosofia/13986-ragione-e-razionalita.html

Carofiglio. (2007). L'arte del dubbio. Palermo: Sallerio.

Cataldi. (2016). Guide di diitto penale. Tratto da Studio Cataldi: https://www.studiocataldi.it/guide-diritto-penale/

Coppola, A. (2017). Come funziona un processo penale. Tratto da La lege per tutti : https://www.laleggepertutti.it/183531_come-funziona-un-processo-penale

D. E. (2018). Derecho Penal. Tratto da Derecho Ecuador: https:// www.derechoecuador.com/penal

Díaz, P. R. (1998). La posición de la intellectio en el sistema retórico clásico. HVMAITAS, 26.

Eire, J. V. (2013). La retórica penal de Jaques Vergès. Tratto da El derecho: http://www.elderecho.com/tribuna/www-elderecho-com/retorica-penal-Jacques-Veres_11_582805002.html

Eire, J. V. (2014). El sistema procesal penal italiano. Tratto da El derecho.com: http://www.elderecho.com/tribuna/penal/sistema_procesal_penal_italiano_11_741055002.html

Elmedi. (2012). Civiltá Greca. Tratto da Pearson Education Library : http://ppbm.elmedi.it/materiali/testifacilitati/v5_st_01.pdf

Española, R. A. (s.d.). Diccionario Real Academia Española. Tratto da Real Academia Española: http://dle.rae.es/

Falero, L. M. (2002). La retórica en el siglo XX. Hacia una Retórica General. DICENDA. Cuadernos de Filología Hispánica. , 25. 
Fornacari, L. d., \& Piemonti, M. G. (2001). Dizionario giuridico: italiano - spagnolo. Milano: Giuffre.

Francesco, A. D. (2015). La logica retorica nel processo penale. Penare il diritto, 18. Tratto da Pensare il diritto: http://www.pensareildiritto. it/wp-content/uploads/2015/09/La-logica-retorica-nel-processopenale.pdf

G., S. (2001). Il luogo ell'oratore. argomentazione topica e retorica forense. Napoli : ESI.

Gamboa, Y. (s.d.). Guía para la escritura del ensayo. Tratto da Pontificia Universidad Catòlica del Ecuador : http://eva.puce.edu.ec/pluginfile.php $/ 161737 / \mathrm{mod}$ _resource/content $/ 0 / \mathrm{Gu} \% \mathrm{C} 3 \% \mathrm{ADa} \% 20 \mathrm{ela}-$ boraci\%C3\%B3n\%20ensayos.pdf

Hechavarria, M. P. (2011). El proceso penal, què es y su principales elementos. Tratto da Gestiopolis: https://www.gestiopolis.com/proceso-penal-que-es-principales-elementos/

López Noreña, G. (2010). La antigua retórica grecoromana y la educación: en la perspectiva de la teoría de la argumentación y la posmodernidad, Edición electrónica gratuita. Texto completo en www. eumed.net/libros/2010d/768/

M., C. (2007). L'arte di comunicare. Milano: Oscar Mondadori.

Mazin, M. (2014). Argomentazione giuridica e retorica forense. Torino: Giappichelli.

Melo, C. (2012). Retorica - Aristoteles. Tratto da Prezi: https:// prezi.com/10dulszyccop/retorica-aristoteles-resumen-capitulo-8-1o-2o-y-3er-libro/

Piazza, F. (2015). Retorica vivente. Un approccio retorico alla filosofia del linguaggio. Iris Unipa, 19.

RAE. (s.d.). Diccionario Real Academia Española. Tratto da http://dle. rae.es/

Ruiz, A. Z. (2007). La Retórica. Razon y Palabra, 50. 
Ruiz, A. Z. (2010). El perfeccionamiento personal a través de la retórica. UMA, 28.

S., S. (1991). L'arte di ottenere ragione. Milano: Adelphi.

Sera, C. D. (s.d.). Dizionario dei sinonimi e contrari. Tratto da Corriere della sera: http://dizionari.corriere.it/dizionario_sinonimi_contrari/P/parlare_1.shtml

Traversi, A. (2014). La difesa penale. Tecniche argomentative e oratorie. Milano: Giuffrè.

Treccani. (s.d.). Dizionario Treccani. Tratto da Treccani : http:// www.treccani.it/vocabolario/dizionario/

Vidas, B. Y. (2017). Aristóteles. Tratto da Bibliografías y Vidas: https://www.biografiasyvidas.com/monografia/aristoteles/

Zorzi, J. P. (2005). Razonamiento jurídico y toma de decisión. Breves notas acerca de la influencia de la racionalidad y la irracionalidad en la decisión judicial. Revista de Filosofía, Derecho y Política, 20.

Recibido: 04/08/2017

Aceptado: $24 / 05 / 2018$

Dennis Valeria Encalada González: Investigadora Jurídica Equimacon S.A.

Correo electrónico: valeriaencalada97@gmail.com 